\title{
A Proof Theoretic View of Constraint Programming
}

\author{
Krzysztof R. Apt* \\ $C W I$ \\ P.O. Box 94079, 1090 GB Amsterdam, The Netherlands \\ and \\ Dept. of Mathematics, Computer Science, Physics \& Astronomy \\ University of Amsterdam, Plantage Muidergracht 24 \\ 1018 TV Amsterdam, The Netherlands \\ aptocwi.nl
}

\begin{abstract}
We provide here a proof theoretic account of constraint programming that attempts to capture the essential ingredients of this programming style. We exemplify it by presenting proof rules for linear constraints over interval domains, and illustrate their use by analyzing the constraint propagation process for the SEND + MORE = MONEY puzzle. We also show how this approach allows one to build new constraint solvers.
\end{abstract}

Keywords: constraints, constraint propagation, proof rules, linear constraints.

\section{Introduction}

\subsection{Motivation}

One of the most interesting recent developments in the area of programming has been constraint programming. A prominent instance of it is constraint logic programming exemplified by such programming languages as $\operatorname{CLP}(\mathcal{R})$, Prolog III or $\mathrm{ECL}^{i} \mathrm{PS}^{e}$. But recently also imperative constraint programming languages emerged, such as 2LP of [17] or CLAIRE of [5]. (For an overview of this area and related references see [27]).

The aim of this paper is to explain the essence of this approach to programming without committing oneself to a particular programming paradigm. We achieve this by providing a

\footnotetext{
*Address for correspondence: CWI, P.O. Box 94079, 1090 GB Amsterdam, The Netherlands
} 
simple proof theoretic framework that allows us in particular to explain constraint propagation, one of the cornerstones of constraint programming.

The simplicity and elegance of constraint logic programming has already led to a general presentation of their operational semantics in [11] that easily can be casted in a proof theoretic jacket. But this account is limited to the logic programming view of constraint programming. Moreover, it treats constraint propagation as a further unexplained atomic action. Admittedly, the latter deficiency has been addressed in [24], where it has been explained how constraint propagation can be defined within the framework of [11].

In our approach we try to "decouple" constraint programming from logic programming by going back to the origins of constraints handling and by viewing computing as a task of transforming one constraint satisfaction problem (CSP) into another, equivalent one. To take into account reasoning by case analysis (that leads to "don't-know" nondeterminism and various forms of backtracking) we further introduce a splitting operation that allows us to split one CSP into two, the union of which is equivalent to the original CSP. The rules that govern this process of transforming one CSP into a finite collection of them fall naturally into four categories and seem to be sufficient to describe the computation process.

By providing such a general view of constraint programming we can use it to analyze the constraint programming process both within the logic programming paradigm and the imperative one. In particular, as we shall see in Sections 3 and 4, we can use it both to study existing constraint solvers and to build new ones. Additionally, as the Appendix shows, we can reason formally about the proposed rules.

As a by-product of these considerations we bring constraint programming closer to the computation as deduction paradigm according to which the computation process is identified with a constructive proof of a formula (a query) from a set of axioms. This paradigm goes back to Herbrand and Gödel and is exemplified by logic programming and functional programming and also by viewing the parsing process as a deduction (see e.g., [21]).

\subsection{Related Work}

A number of papers have advocated theorem proving as a means to account for various aspects constraint logic programming. In particular, in [8] a Gentzen-style sequent calculus was used to develop a logical semantics of constraint logic programs and in [1] proof-theoretic techniques were applied to compare the intended theory and its actual implementation for various constraint logic programming systems.

Next, a proof theoretic approach to constraint propagation within the constraint logic programming framework has been proposed in [10]. In this work so-called constraint handling rules (CHR) have been introduced. CHR are available as part of the $\mathrm{ECL}^{i} \mathrm{PS}^{e}$ system and allow the user to define his/her own constraint solvers.

Further, a related to ours approach to constraint programming has been proposed in [28]. In this work constraint logic programs are identified with so-called if-and-only-if definitions augmented with integrity constraints. The if-and-only-if definitions are used to define the "usual" 
logic programming computation step while the integrity constraints are used to account for the constraint propagation process that is identified with a complete resolution strategy. This approach is further elaborated and generalized in [13].

Our view of constraint programming is also compatible with that expounded in [23] where constraint programming is presented without a commitment to a specific programming paradigm. In fact, our approach allows one to couch his concepts of propagators, inference engines and distributors into a more specific, proof theoretic, framework.

The approach here presented is closest to the one introduced independently in [6]. Even though the overall objectives are essentially the same, the emphasis in his paper lies rather on defining specific techniques of constraint programming such as arc consistency and forward checking by means of proof rules and strategies.

Finally, let us mention the following unsubstantiated remark that we found in $[16$, page 1115]: "In fact, virtually any form of constraint propagation can be defined in terms of rules of inference".

\subsection{Preliminaries}

We recall here the relevant definitions. Consider a finite sequence of variables $\mathcal{X}:=x_{1}, \ldots, x_{n}$ where $n \geq 0$, with respective domains $\mathcal{D}:=D_{1}, \ldots, D_{n}$ associated with them. So each variable $x_{i}$ ranges over the domain $D_{i}$. By a constraint $C$ on $\mathcal{X}$ we mean a subset of $D_{1} \times \ldots \times D_{n}$. If $C$ equals $D_{1} \times \ldots \times D_{n}$ then we say that $C$ is solved. In the boundary case when the number $n$ of the variables equals 0 we admit two constraints, denoted by $T$ and $\perp$, that denote respectively the true constraint (for example $0=0$ ) and the false constraint (for example $0=1$ ).

By a constraint satisfaction problem, CSP in short, we mean a triple $\langle\mathcal{X}, \mathcal{D}, \mathcal{C}\rangle$, where $\mathcal{C}$ is a finite set of constraints, each on a subsequence of $\mathcal{X}$.

Given a $\operatorname{CSP}\langle\mathcal{X}, \mathcal{D}, \mathcal{C}\rangle$ with $\mathcal{X}:=x_{1}, \ldots, x_{n}$ and $\mathcal{D}:=D_{1}, \ldots, D_{n}$, we say that an $n$-tuple $\left(d_{1}, \ldots, d_{n}\right) \in D_{1} \times \ldots \times D_{n}$ is a solution to $\langle\mathcal{X}, \mathcal{D}, \mathcal{C}\rangle$ if for every constraint $C \in \mathcal{C}$ on a sequence $x_{i_{1}}, \ldots, x_{i_{m}}$ of the variables from $\mathcal{X}$ we have

$$
\left(d_{i_{1}}, \ldots, d_{i_{m}}\right) \in C
$$

Below we represent a $\operatorname{CSP}\langle\mathcal{X}, \mathcal{D}, \mathcal{C}\rangle$ as an expression of the form $\langle\mathcal{C} ; \mathcal{D} \mathcal{E}\rangle$, where $\mathcal{D} \mathcal{E}:=$ $\left\{x_{1} \in D_{1}, \ldots, x_{n} \in D_{n}\right\}$. We call a construct of the form $x \in D$ a domain expression. We stress the fact that a domain expression is not a constraint. By considering domain expressions separately, we can focus in the sequel our attention on the proof rules that reduce domains. Such rules are very common when dealing with linear constraints and constraints on reals.

An alternative approach that we did not pursue here, is to dispense with the domains by viewing each constraint as an $n$-ary relation and by associating with each domain a unary constraint that coincides with it. In this approach study of domain reduction becomes artificial.

To simplify the notation from now on we omit the " \{\} " brackets when presenting $\mathcal{C}$ and $\mathcal{D E}$.

We call a CSP solved if it is of the form $\langle\emptyset ; \mathcal{D E}\rangle$ where no domain in $\mathcal{D E}$ is empty, and failed if it either contains the false constraint $\perp$ or some of its domains is empty. So a failed 
CSP admits no solution.

Given two CSP's $\phi$ and $\psi$, we call $\phi$ a variant of $\psi$ if the removal of solved constraints from $\phi$ and $\psi$ yields the same CSP.

In what follows we assume that the constraints and the domain expressions are defined in some specific, further unspecified, language. In this representation of the constraints it is implicit that each of them is a subset of the Cartesian product of the associated variable domains. For example, if we consider the $\operatorname{CSP}\langle x<y ; x \in[0 . .10], y \in[5 . .10]\rangle$, then we view the constraint $x<y$ as the set $\{(a, b) \mid a \in[0 . .10], b \in[5 . .10], a<b\}$.

Given a constraint $c$ on the variables $x_{1}, \ldots, x_{n}$ with respective domains $D_{1}, \ldots, D_{n}$, and a sequence of domains $D_{1}^{\prime}, \ldots, D_{n}^{\prime}$ such that for $i \in[1 . . n]$ we have $D_{i}^{\prime} \subseteq D_{i}$, we say that $c^{\prime}$ is the result of restricting $c$ to the domains $D_{1}^{\prime}, \ldots, D_{n}^{\prime}$ if $c^{\prime}=c \cap\left(D_{1}^{\prime} \times \ldots \times D_{n}^{\prime}\right)$.

\section{The Proof Theoretic Framework}

In this section we introduce a proof theoretic framework that will be used throughout the paper.

\subsection{Format of the Proof Rules}

In what follows we consider two types of proof rules that we call deterministic and splitting. The deterministic rules are of the form

$$
\frac{\phi}{\psi}
$$

where $\phi$ and $\psi$ are CSP's. We assume here that $\phi$ is not failed and its set of constraints is non-empty. Depending on the form of the conclusion $\psi$ we distinguish two cases. Assume that

$$
\phi:=\langle\mathcal{C} ; \mathcal{D E}\rangle
$$

and

$$
\psi:=\left\langle\mathcal{C}^{\prime} ; \mathcal{D} \mathcal{E}^{\prime}\right\rangle
$$

- Domain reduction rules, or in short reduction rules. These are rules in which the new domains are respective subsets of the old domains and the new constraints are respective restrictions of the old constraints to the new domains.

So here $\mathcal{D E}:=x_{1} \in D_{1}, \ldots, x_{n} \in D_{n}, \mathcal{D} \mathcal{E}^{\prime}:=x_{1} \in D_{1}^{\prime}, \ldots, x_{n} \in D_{n}^{\prime}$, for $i \in[1 . . n]$ we have $D_{i}^{\prime} \subseteq D_{i}$, and $\mathcal{C}^{\prime}$ is the result of restricting each constraint in $\mathcal{C}$ to the corresponding subsequence of the domains $D_{1}^{\prime}, \ldots, D_{n}^{\prime}$.

Here a failure is reached only when a domain of one or more variables gets reduced to the empty set.

When all constraints in $\mathcal{C}^{\prime}$ are solved, we call such a rule a solving rule. 
- Transformation rules. These rules are not domain reduction rules and are such that $\mathcal{C}^{\prime} \neq \emptyset$ and $\mathcal{D E} \subseteq \mathcal{D} \mathcal{E}^{\prime}$.

The inclusion between $\mathcal{D E}$ and $\mathcal{D} \mathcal{E}^{\prime}$ means that the domains of common variables are identical and that possibly new domain expressions have been added to $\mathcal{D E}$. Such new domain expressions deal with new variables on which some constraints have been introduced.

Here a failure is reached only when the false constraint is generated.

The splitting rules are of the form

$$
\frac{\phi}{\psi_{1} \mid \psi_{2}}
$$

where $\phi, \psi_{1}$ and $\psi_{2}$ are CSP's. As for deterministic rules we assume here that $\phi$ is not failed and its set of constraints is non-empty. In what follows we only consider splitting rules in which $\phi, \psi_{1}$ and $\psi_{2}$ are CSP's with the same sequence of variables.

These rules allow us to replace one CSP by two CSP's. The intuition is that their "union" is "equivalent" to the original CSP. They are counterparts of the rules just introduced. So, again, we distinguish two cases.

- Reduction splitting rules. These are rules such that both $\frac{\psi}{\phi_{1}}$ and $\frac{\psi}{\phi_{2}}$ are reduction rules.

- Transformation splitting rules. These are rules such that both $\frac{\psi}{\phi_{1}}$ and $\frac{\psi}{\phi_{2}}$ are transformation rules.

\subsection{Examples of Proof Rules}

In the sequel when presenting specific proof rules we delete from the conclusion all solved constraints. Also, we abbreviate the domain expression $x \in\{a\}$ to $x=a$.

As an example of a reduction rule consider the following rule:

$$
\begin{gathered}
\text { EQUALITY } 1 \\
\frac{\left\langle x=y ; x \in D_{1}, y \in D_{2}\right\rangle}{\left\langle x=y ; x \in D_{1} \cap D_{2}, y \in D_{1} \cap D_{2}\right\rangle}
\end{gathered}
$$

Note that this rule yields a failure when $D_{1} \cap D_{2}=\emptyset$. In case $D_{1} \cap D_{2}$ is a singleton this rule becomes a solving rule, that is the constraint $x=y$ becomes solved (and hence deleted). Note also the following solving rule:

$$
\begin{aligned}
& \text { EQUALITY } 2 \\
& \frac{\langle x=x ; x \in D\rangle}{\langle; x \in D\rangle}
\end{aligned}
$$

Following the just introduced convention we dropped the constraint from the conclusion of the EQUALITY 2 rule. This explains its format.

As further examples of solving rules consider the following three concerning disequality: 


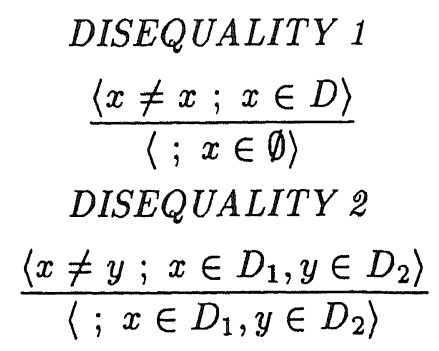

where $D_{1} \cap D_{2}=\emptyset$,

$$
\begin{gathered}
\text { DISEQUALITY } 3 \\
\frac{\langle x \neq y ; x \in D, y=a\rangle}{\langle; x \in D-\{a\}, y=a\rangle}
\end{gathered}
$$

where $a \in D$, and similarly with $x \neq y$ replaced by $y \neq x$.

So the DISEQUALITY 1 rule yields a failure while the DISEQUALITY 3 rule can yield a failure.

Next, as an example of a transformation rule consider the following rule that substitutes a variable by a value:

$$
\begin{gathered}
\text { SUBSTITUTION } \\
\langle\mathcal{C} ; \mathcal{D} \mathcal{E}, x=a\rangle \\
\hline\langle\mathcal{C}\{x / \bar{a}\} ; \mathcal{D E}, x=a\rangle
\end{gathered}
$$

where $x$ occurs in $\mathcal{C}$.

Here $\bar{a}$ stands for the constant that denotes in the underlying language the value $a$ and $\mathcal{C}\{x / \bar{a}\}$ denotes the set of constraints obtained from $\mathcal{C}$ by substituting in it every occurrence of $x$ by $\bar{a}$. So $x$ does not occur in $\mathcal{C}\{x / \bar{a}\}$.

Another example of a transformation rule forms the following rule:

\section{DELETION}

$$
\frac{\langle\mathcal{C} \cup\{T\} ; \mathcal{D E}\rangle}{\langle\mathcal{C} ; \mathcal{D E}\rangle}
$$

Let us consider now the splitting rules. A natural class of examples of reduction splitting rules form domain splitting rules. These are rules of the form:

$$
\frac{\langle\mathcal{C} ; \mathcal{D E}, x \in D\rangle}{\left\langle\mathcal{C}^{\prime} ; \mathcal{D E}, x \in D_{1}\right\rangle \mid\left\langle\mathcal{C}^{\prime \prime} ; \mathcal{D E}, x \in D_{2}\right\rangle}
$$

where $D_{1} \cup D_{2}=D, D_{i} \neq \emptyset$ for $i \in\{1,2\}, \mathcal{C}^{\prime}$ is the result of restricting each constraint in $\mathcal{C}$ to the corresponding subsequence of the domains in $\mathcal{D E}$ and $D_{1}$, and analogously with $\mathcal{C}^{\prime \prime}$.

If such a rule does not depend on $\mathcal{C}$ and $\mathcal{D E}$ we abbreviate it to

$$
\frac{x \in D}{x \in D_{1} \mid x \in D_{2}}
$$

Two specific instances are: 


\section{ENUMERATION}

$$
\frac{x \in D}{x=a \mid x \in D-\{a\}}
$$

where $D$ is a finite domain with at least two elements and $a \in D$, and

$$
\begin{gathered}
\text { BISECTION } \\
\frac{x \in[a . . b]}{x \in\left[a . . \frac{a+b}{2}\right] \mid x \in\left[\frac{a+b}{2} . . b\right]}
\end{gathered}
$$

where $[a . . b]$ a closed non-empty interval of reals. Here we wish to preserve the property that the intervals are closed so the new intervals are not disjoint.

Finally, a natural class of examples of transformation splitting rules form constraint splitting rules. They have the following form:

$$
\frac{\langle\mathcal{C}, C ; \mathcal{D E}\rangle}{\left\langle\mathcal{C}, C_{1} ; \mathcal{D E}\right\rangle \mid\left\langle\mathcal{C}, C_{2} ; \mathcal{D E}\right\rangle}
$$

where

- every solution to $\langle\mathcal{C}, C ; \mathcal{D E}\rangle$ is a solution to $\left\langle\mathcal{C}, C_{1} ; \mathcal{D E}\right\rangle$ or $\left\langle\mathcal{C}, C_{2} ; \mathcal{D E}\right\rangle$,

- every solution to $\left\langle\mathcal{C}, C_{i} ; \mathcal{D E}\right\rangle(i \in[1,2])$ is a solution to $\langle\mathcal{C}, C ; \mathcal{D E}\rangle$.

If such a rule does not depend on $\mathcal{C}$ and $\mathcal{D E}$ we abbreviate it to

$$
\frac{C}{C_{1} \mid C_{2}}
$$

A particular instance is:

$$
\frac{|x-y|=a}{x-y=a \mid x-y=-a}
$$

where $x$ and $y$ are integer variables and $a$ is an integer.

\subsection{Derivations}

Now that we have defined the proof rules, we define the result of applying a proof rule to a CSP. Assume a CSP of the form $\left\langle\mathcal{C} \cup \mathcal{C}_{1} ; \mathcal{D} \cup \mathcal{D}_{1}\right\rangle$. First, consider a deterministic rule, so a rule of the form

$$
\frac{\left\langle\mathcal{C}_{1} ; \mathcal{D}_{1}\right\rangle}{\left\langle\mathcal{C}_{2} ; \mathcal{D}_{2}\right\rangle}
$$

Here a clash of variables can take place if some variable of $\mathcal{C}_{2}$ also appears in $\mathcal{C}$ but not in $\mathcal{C}_{1}$. Then such a variable of $\mathcal{C}_{2}$ should be renamed first. So let us rename the variables of $\left\langle\mathcal{C}_{2} ; \mathcal{D}_{2}\right\rangle$ that appear in $\mathcal{C}$ but not in $\mathcal{C}_{1}$ by some fresh variables and denote the so obtained CSP by $\left\langle\mathcal{C}_{2}^{\prime} ; \mathcal{D}_{2}^{\prime}\right\rangle$. 
We say that rule (1) can be applied to $\left\langle\mathcal{C} \cup \mathcal{C}_{1} ; \mathcal{D} \cup \mathcal{D}_{1}\right\rangle$ and call

$$
\left\langle\mathcal{C} \cup \mathcal{C}_{2}^{\prime} ; \mathcal{D} \cup \mathcal{D}_{2}^{\prime}\right\rangle
$$

the result of applying rule (1) to $\left\langle\mathcal{C} \cup \mathcal{C}_{1} ; \mathcal{D} \cup \mathcal{D}_{1}\right\rangle$. If $\left\langle\mathcal{C} \cup \mathcal{C}_{2}^{\prime} ; \mathcal{D} \cup \mathcal{D}_{2}^{\prime}\right\rangle$ is not a variant of $\left\langle\mathcal{C} \cup \mathcal{C}_{1} ; \mathcal{D} \cup \mathcal{D}_{1}\right\rangle$, then we say that it is the result of a relevant application of rule (1) to $\left\langle\mathcal{C} \cup \mathcal{C}_{1} ; \mathcal{D} \cup \mathcal{D}_{1}\right\rangle$

Further, given a CSP $\phi$ and a deterministic rule $R$, we say that $\phi$ is closed under the applications of $R$ if either $R$ cannot be applied to $\phi$ or no application of it to $\phi$ is relevant.

For example, assume for a moment the expected interpretation of propositional formulas and consider the CSP $\phi:=\langle x \wedge y=z ; x=1, y=0, z=0\rangle$. Here $x=1$ is an abbreviation for the domain expression $x \in\{1\}$ and similarly for the other variables.

This CSP is closed under the applications of the transformation rule

$$
\frac{\left\langle x \wedge y=z ; x=1, y \in D_{y}, z \in D_{z}\right\rangle}{\left\langle z=y ; x=1, y \in D_{y}, z \in D_{z}\right\rangle}
$$

Indeed, this rule can be applied to $\phi$; the outcome is $\psi:=\langle z=y ; x=1, y=0, z=0\rangle$. After the removal of solved constraints from $\phi$ and $\psi$ we get in both cases the solved CSP $\langle\emptyset ; x=1, y=0, z=0\rangle$.

In contrast, the CSP $\phi:=\langle x \wedge y=z ; x=1, y \in\{0,1\}, z \in\{0,1\}\rangle$ is not closed under the applications of the above rule because $\langle z=y ; x=1, y \in\{0,1\}, z \in\{0,1\}\rangle$ is not a variant of $\phi$.

Next, consider a splitting rule, so a rule of the form

$$
\frac{\left\langle\mathcal{C}_{1} ; \mathcal{D}_{1}\right\rangle}{\left\langle\mathcal{C}_{2} ; \mathcal{D}_{2}\right\rangle \mid\left\langle\mathcal{C}_{3} ; \mathcal{D}_{3}\right\rangle}
$$

We then say that rule (2) can be applied to $\left\langle\mathcal{C} \cup \mathcal{C}_{1} ; \mathcal{D} \cup \mathcal{D}_{1}\right\rangle$ and call

$$
\left\langle\mathcal{C} \cup \mathcal{C}_{2} ; \mathcal{D} \cup \mathcal{D}_{2}\right\rangle \mid\left\langle\mathcal{C} \cup \mathcal{C}_{3} ; \mathcal{D} \cup \mathcal{D}_{3}\right\rangle
$$

the result of applying it to $\left\langle\mathcal{C} \cup \mathcal{C}_{1} ; \mathcal{D} \cup \mathcal{D}_{1}\right\rangle$. If neither $\left\langle\mathcal{C} \cup \mathcal{C}_{2} ; \mathcal{D} \cup \mathcal{D}_{2}\right\rangle$ nor $\left\langle\mathcal{C} \cup \mathcal{C}_{3} ; \mathcal{D} \cup \mathcal{D}_{3}\right\rangle$ is a variant of $\left\langle\mathcal{C} \cup \mathcal{C}_{1} ; \mathcal{D} \cup \mathcal{D}_{1}\right\rangle$, then we say that (3) is the result of a relevant application of rule (2) to $\left\langle\mathcal{C} \cup \mathcal{C}_{1} ; \mathcal{D} \cup \mathcal{D}_{1}\right\rangle$. (Recall that by assumption all three CSP's $\left\langle\mathcal{C}_{i} ; \mathcal{D}_{i}\right\rangle$, where $i \in[1 . .3]$, have the same sequence of variables, so we do not need to worry here about variable clashes.)

Finally, we introduce the notions of a proof tree and of a derivation.

Definition 2.1. Assume a set of proof rules. A proof tree is a tree the nodes of which are CSP's. Further, each node has at most two direct descendants and for each node $\phi$ the following holds:

- If $\phi$ is a leaf, then no application of a rule to $\psi$ is relevant;

- If $\phi$ has precisely one direct descendant, say $\psi$, then $\psi$ is the result of a relevant application of a proof rule to $\phi$; 
- If $\phi$ has precisely two direct descendants, say $\psi_{1}$ and $\psi_{2}$, then $\psi_{1} \mid \psi_{2}$ is the result of a relevant application of a proof rule to $\phi$.

A derivation is a branch in a proof tree. A derivation is called successful if it is finite and its last element is a solved CSP. A derivation is called failed if it is finite and its last element is a failed CSP.

The idea behind the above definition is that we consider in the proof trees only those applications of the proof rules that cause some change. Note also that more proof rules can be applicable to a given CSP, so a specific CSP can be a root of several proof trees.

Note that some finite derivations are neither successful nor failed. In fact, many constraint solvers yield CSP's that are neither solved nor failed - their aim is to bring the initial CSP to some specific, simpler form.

In some cases this third possibility does not arise. Indeed, consider a non-failed CSP with a non-empty set of constraints on finite domains. Then either the DELETION or the SUBSTITUTION or the ENUMERATION rule can be applied to it and moreover each such application is always relevant. So in presence of the above three rules for CSP's with finite domains each finite derivation is either successful or failed.

\subsection{Equivalent CSP's}

We introduced the proof rules so that we can reduce one CSP to another CSP or to two CSP's, which are in some sense "smaller" yet "equivalent". Both notions can be made precise but the first one will not play any role in our considerations, so we only present an adequate notion of equivalence. Because the considered proof rules are of a specific form, we limit ourselves in the definition to specific pairs of CSP's.

Definition 2.2. Consider two CSP's $\phi$ and $\psi$ such that all variables of $\phi$ are also present in $\psi$. We say that the CSP's $\phi$ and $\psi$ are equivalent if

- every solution to $\phi$ is or can be extended to a solution to $\psi$,

- for every solution to $\psi$ its restriction to the variables of $\phi$ is a solution to $\phi$.

In particular, two CSP's with the same sequence of variables are equivalent if they have the same set of solutions. So for example the CSP's

$$
\langle 3 x-5 y=4 ; x \in[0 . .9], y \in[1 . .8]\rangle
$$

and

$$
\langle 3 x-5 y=4 ; x \in[3 . .8], y \in[1 . .4]\rangle
$$

are equivalent, since both of them have $x=3, y=1$ and $x=8, y=4$ as the only solutions, and, for $\mathcal{D} \mathcal{E}:=x \in D_{x}, y \in D_{y}, z \in D_{z}$, so are

$$
\langle x<y, y<z ; \mathcal{D E}\rangle
$$


and

$$
\langle x<y, y<z, x<z ; \mathcal{D E}\rangle \text {. }
$$

In contrast,

$$
\left\langle x<z ; x \in D_{x}, z \in D_{z}\right\rangle
$$

and

$$
\langle x<y, y<z ; \mathcal{D E}\rangle
$$

are not equivalent, as not each solution to the former extends to a solution of the latter.

This brings us to the following notion where we make use of the fact that the considered proof rules are of a specific form.

\section{Definition 2.3.}

(i) A proof rule

$$
\frac{\phi}{\psi}
$$

is called equivalence preserving if $\phi$ and $\psi$ are equivalent.

(ii) A proof rule

$$
\frac{\phi}{\psi_{1} \mid \psi_{2}}
$$

is called equivalence preserving if

- every solution to $\phi$ is a solution to $\psi_{1}$ or to $\psi_{2}$,

- every solution to $\psi_{i}(i \in[1,2])$ is a solution to $\phi$.

All the rules discussed so far are equivalence preserving. From the way we introduce the proof rules in the sequel it will be clear that all of them are also equivalence preserving.

This completes the presentation of our proof theoretic framework. In order to use it to model constraint programming the proof rules above introduced have to be "customized" to a specific language in which constraints are defined and to specific domains. In what follows we present an example of such a customization that deals with linear constraints over interval and finite domains.

Such rules should be selected and scheduled in an appropriate way and some strategy should be employed to traverse the generated proof trees. We defer discussion of these issues to Subsection 5.2. 


\section{Linear Constraints over Interval Domains}

In this section we consider linear constraints over interval domains. We use the introduced rules to discuss the behaviour of the ECL ${ }^{i} \mathrm{PS}^{e}$ finite domain solver and, by means of an example, to analyse the $S E N D+M O R E=M O N E Y$ puzzle.

First, let us recall the relevant definitions. By a linear expression we mean a term in the language that contains two constants 0 and 1 , the unary minus function - and two binary functions + and - , both written in the infix notation. We abbreviate terms of the form

to $n$, terms of the form

$$
\underbrace{1+\ldots+1}_{n \text { times }}
$$

$$
\underbrace{x+\ldots+x}_{n \text { times }}
$$

to $n x$ and analogously with -1 and $-x$ used instead of 1 and $x$. So (using appropriate transformation rules) each linear expression can be equivalently written in the form

$$
a_{1} x_{1}+\ldots+a_{n} x_{n}+a_{n+1}
$$

where $n \geq 0, a_{1}, \ldots, a_{n}$ are non-zero integers, $x_{1}, \ldots, x_{n}$ are different variables and $a_{n+1}$ is an integer.

By a linear constraint we mean a formula of the form

$$
s \text { opt }
$$

where $s$ and $t$ are linear expressions and $o p \in\{<, \leq,=, \neq, \geq,>\}$. In what follows we drop the qualification "linear" when discussing linear expressions and linear constraints.

Further, we call

- $s<t$ and $s>t$ strict inequality constraints,

- $s \leq t$ and $s \geq t$ inequality constraints,

- $s=t$ an equality constraint,

- $s \neq t$ a disequality constraint,

- $x \neq y$, for variables $x, y$, a simple disequality constraint.

By an integer interval, or an interval in short, we mean an expression of the form

$$
[a . . b]
$$

where $a$ and $b$ are integers; $[a . . b]$ denotes the set of all integers between $a$ and $b$, including $a$ and $b$. If $a>b$, we call [a..b] the empty interval.

Finally, by a range we mean an expression of the form

$$
x \in I
$$

where $x$ is a variable and $I$ is an interval. We abbreviate $x \in[a . . b]$ to $x=a$ if $a=b$ and write $x \in \emptyset$ if $a>b$.

In what follows we discuss various rules that allow us to manipulate linear constraints over interval domains. We assume that all considered linear constraints have at least one variable. 


\subsection{Reduction Rules for Inequality Constraints}

We begin with the inequality constraints. Using appropriate transformation rules each inequality constraint can be equivalently written in the form

$$
\sum_{i \in P O S} a_{i} x_{i}-\sum_{i \in N E G} a_{i} x_{i} \leq b
$$

where

- $a_{i}$ is a positive integer for $i \in P O S \cup N E G$,

- $x_{i}$ and $x_{j}$ are different variables for $i \neq j$ and $i, j \in P O S \cup N E G$,

- $b$ is an integer.

Assume the ranges

$$
x_{i} \in\left[l_{i} . . h_{i}\right]
$$

for $i \in P O S \cup N E G$.

Choose now some $j \in P O S$ and let us rewrite (4) as

$$
x_{j} \leq \frac{b-\sum_{i \in P O S-\{j\}} a_{i} x_{i}+\sum_{i \in N E G} a_{i} x_{i}}{a_{j}}
$$

Computing the maximum of the expression on the right-hand side w.r.t. the ranges of the involved variables we get

$$
x_{j} \leq \alpha_{j}
$$

where

$$
\alpha_{j}:=\frac{b-\sum_{i \in P O S-\{j\}} a_{i} l_{i}+\sum_{i \in N E G} a_{i} h_{i}}{a_{j}}
$$

so, since the variables assume integer values,

$$
x_{j} \leq\left\lfloor\alpha_{j}\right\rfloor
$$

We conclude that

$$
x_{j} \in\left[l_{j} . . \min \left(h_{j},\left\lfloor\alpha_{j}\right\rfloor\right)\right] .
$$

By analogous calculations we conclude for $j \in N E G$

$$
x_{j} \geq\left\lceil\beta_{j}\right\rceil
$$

where

$$
\beta_{j}:=\frac{-b+\sum_{i \in P O S} a_{i} l_{i}-\sum_{i \in N E G-\{j\}} a_{i} h_{i}}{a_{j}}
$$

In this case we conclude that

$$
x_{j} \in\left[\max \left(l_{j},\left\lceil\beta_{j}\right\rceil\right) . . h_{j}\right] .
$$

This brings us to the following reduction rule for inequality constraints: 


$$
\frac{\left\langle\sum_{i \in P O S} a_{i} x_{i}-\sum_{i \in N E G} a_{i} x_{i} \leq b ; x_{1} \in\left[l_{1} . . h_{1}\right], \ldots, x_{n} \in\left[l_{n} . . h_{n}\right]\right\rangle}{\left\langle\sum_{i \in P O S} a_{i} x_{i}-\sum_{i \in N E G} a_{i} x_{i} \leq b ; x_{1} \in\left[l_{1}^{\prime} . . h_{1}^{\prime}\right], \ldots, x_{n} \in\left[l_{n}^{\prime} . . h_{n}^{\prime}\right]\right\rangle}
$$

where for $j \in P O S$

$$
l_{j}^{\prime}:=l_{j}, h_{j}^{\prime}:=\min \left(h_{j},\left\lfloor\alpha_{j}\right\rfloor\right)
$$

and for $j \in N E G$

$$
l_{j}^{\prime}:=\max \left(l_{j},\left\lceil\beta_{j}\right\rceil\right), h_{j}^{\prime}:=h_{j} .
$$

\subsection{Reduction Rules for Equality Constraints}

Each equality constraint can be equivalently written as two inequality constraints. By combining the corresponding reduction rules for these two inequality constraints we obtain a reduction rule for an equality constraint. More specifically, each equality constraint can be equivalently written in the form

$$
\sum_{i \in P O S} a_{i} x_{i}-\sum_{i \in N E G} a_{i} x_{i}=b
$$

where we adopt the conditions that follow (4) in the previous subsection.

Assume now the ranges

$$
x_{1} \in\left[l_{1} . . h_{1}\right], \ldots, x_{n} \in\left[l_{n} . . h_{n}\right] .
$$

We infer then both the conclusion of the LINEAR INEQUALITY 1 reduction rule and

$$
x_{1} \in\left[l_{1}^{\prime \prime} . . h_{1}^{\prime \prime}\right], \ldots, x_{n} \in\left[l_{n}^{\prime \prime} . h_{n}^{\prime \prime}\right]
$$

where for $j \in P O S$

$$
l_{j}^{\prime \prime}:=\max \left(l_{j},\left\lceil\gamma_{j}\right\rceil\right), h_{j}^{\prime \prime}:=h_{j}
$$

with

$$
\gamma_{j}:=\frac{b-\sum_{i \in P O S-\{j\}} a_{i} h_{i}+\sum_{i \in N E G} a_{i} l_{i}}{a_{j}}
$$

and for $j \in N E G$

$$
l_{j}^{\prime \prime}:=l_{j}, h_{j}^{\prime \prime}:=\min \left(h_{j},\left\lfloor\delta_{j}\right\rfloor\right)
$$

with

$$
\delta_{j}:=\frac{-b+\sum_{i \in P O S} a_{i} h_{i}-\sum_{i \in N E G-\{j\}} a_{i} l_{i}}{a_{j}}
$$

This yields the following reduction rule:

\section{LINEAR EQUALITY}

$$
\frac{\left\langle\sum_{i \in P O S} a_{i} x_{i}-\sum_{i \in N E G} a_{i} x_{i}=b ; x_{1} \in\left[l_{1} . . h_{1}\right], \ldots, x_{n} \in\left[l_{n} . . h_{n}\right]\right\rangle}{\left\langle\sum_{i \in P O S} a_{i} x_{i}-\sum_{i \in N E G} a_{i} x_{i}=b ; x_{1} \in\left[l_{1}^{\prime} . . h_{1}^{\prime}\right], \ldots, x_{n} \in\left[l_{n}^{\prime} . . h_{n}^{\prime}\right]\right\rangle}
$$


where for $j \in P O S$

$$
l_{j}^{\prime}:=\max \left(l_{j},\left\lceil\gamma_{j}\right\rceil\right), h_{j}^{\prime}:=\min \left(h_{j},\left\lfloor\alpha_{j}\right\rfloor\right)
$$

and for $j \in N E G$

$$
l_{j}^{\prime}:=\max \left(l_{j},\left\lceil\beta_{j}\right\rceil\right), h_{j}^{\prime}:=\min \left(h_{j},\left\lfloor\delta_{j}\right\rfloor\right) .
$$

As an example of the use of the above reduction rule consider the CSP $\langle 3 x-5 y=4 ; x \in$ $[0 . .9], y \in[1 . .8]\rangle$. A straightforward calculation shows that $x \in[3 . .9], y \in[1 . .4]$ are the ranges in the conclusion of LINEAR EQUALITY rule. Another application of the rule yields the ranges $x \in[3 . .8]$ and $y \in[1 . .4]$ upon which the process stabilizes.

Note that if in (5) there is only one variable, the LINEAR EQUALITY rule reduces to the following solving rule:

$$
\frac{\langle a x=b ; x \in[l . . h]\rangle}{\left\langle; x \in\left\{\frac{b}{a}\right\} \cap[l . . h]\right\rangle}
$$

So, if $a$ divides $b$ and $l \leq \frac{b}{a} \leq h$, the domain expression $x=\frac{b}{a}$ is inferred, and otherwise a failure is reached.

\subsection{Transformation Rules for Inequality and Equality Constraints}

The above reduction rules can be applied only to inequality and equality constraints that are in a specific form, (4) or (5). So we need to augment the introduced reduction rules by appropriate transformation rules. Depending on the level of description one can content oneself with a couple of general rules or several very detailed ones. These rules are pretty straightforward and are omitted.

\subsection{Rules for Disequality Constraints}

The reduction rules for simple disequalities are very natural. First, note that the following rule

$$
\begin{aligned}
& \text { SIMPLE DISEQUALITY } 1 \\
& \frac{\langle x \neq y ; x \in[a . . b], y \in[c . . d]\rangle}{\langle; x \in[a . . b], y \in[c . . d]\rangle}
\end{aligned}
$$

where $b<c$ or $d<a$, is an instance of the DISEQUALITY 2 solving rule introduced in Subsection 2.2 and where following the convention there mentioned we dropped the constraint and the domain expressions from the conclusion of the proof rule.

Next, we adopt the following two solving rules that are instances of the solving DISEQUALITY 3 rule:

\section{SIMPLE DISEQUALITY 2}

$$
\frac{\langle x \neq y ; x \in[a . . b], y=a\rangle}{\langle; x \in[a+1 . . b], y=a\rangle}
$$




$$
\begin{gathered}
\text { SIMPLE DISEQUALITY } 3 \\
\frac{\langle x \neq y ; x \in[a . . b], y=b\rangle}{\langle; x \in[a . . b-1], y=b\rangle}
\end{gathered}
$$

and similarly with $x \neq y$ replaced by $y \neq x$. Recall that the domain expression $y=a$ is a shorthand for $y \in[a . . a]$.

To deal with disequality constraints that are not simple ones we use the following notation. Given a linear expression $s$ and a sequence of ranges involving all the variables of $s$ we denote by $s^{-}$the minimum $s$ can take w.r.t. these ranges and by $s^{+}$the maximum $s$ can take w.r.t. these ranges. The considerations of Subsection 3.1 show how $s^{-}$and $s^{+}$can be computed.

We now introduce the following transformation rule for non-simple disequality constraints:

$$
\begin{gathered}
\text { DISEQUALITY } 3 \\
\langle s \neq t ; \mathcal{D E}\rangle \\
\frac{\left\langle s \neq t, x=s ; x \in\left[s^{-} . . s^{+}\right], \mathcal{D E}\right\rangle}{\langle x \neq t}
\end{gathered}
$$

where

- $s$ is not a variable,

- $x$ is a fresh variable,

- $\mathcal{D E}$ is a sequence of the ranges involving the variables present in $s$ and $t$,

- $s^{-}$and $s^{+}$are computed w.r.t. the ranges in $\mathcal{D E}$.

An analogous rule is introduced for the inequality $s \neq t$, where $t$ is not a variable.

\subsection{Domain splitting rules}

We conclude our treatment of linear constraints over interval domains by presenting three specific domain splitting rules. Because at the moment the assumed domains are intervals, these rules so designed that the domains remain intervals.

$$
\begin{aligned}
& \text { INTERVAL SPLITTING } 1 \\
& \frac{x \in[a . . b]}{x=a \mid x \in[a+1 . . b]}
\end{aligned}
$$

where $a<b$,

$$
\begin{aligned}
& \text { INTERVAL SPLITTING } 2 \\
& \frac{x \in[a . . b]}{x=b \mid x \in[a . . b-1]}
\end{aligned}
$$

where $a<b$,

$$
\begin{gathered}
\text { INTERVAL SPLITTING } 3 \\
\langle x \neq c ; x \in[a . . b]\rangle \\
\langle; x \in[a . . c-1]\rangle \mid\langle; x \in[c+1 . . b]\rangle
\end{gathered}
$$

where $a<c<b$.

Finally, to deal with the strict inequality constraints it suffices to use expected transformation rules that reduce them to inequalities and disequalities. 


\subsection{Shifting from Intervals to Finite Domains}

In our presentation we took care that all the rules preserved the property that the domains are intervals. In some systems, such as ECL ${ }^{i} \mathrm{PS}^{e}$, this property is relaxed and instead of finite intervals finite sets of integers are chosen. To model the use of such finite domains it suffices to modify some of the rules introduced above.

In the case of inequality constraints we can use the following minor modification of the LINEAR INEQUALITY 1 reduction rule:

$$
\begin{gathered}
\text { LINEAR INEQUALITY } 2 \\
\frac{\left\langle\sum_{i \in P O S} a_{i} x_{i}-\sum_{i \in N E G} a_{i} x_{i} \leq b ; x_{1} \in D_{1}, \ldots, x_{n} \in D_{n}\right\rangle}{\left\langle\sum_{i \in P O S} a_{i} x_{i}-\sum_{i \in N E G} a_{i} x_{i} \leq b ; x_{1} \in\left[l_{1}^{\prime} . . h_{1}^{\prime}\right] \cap D_{1}, \ldots, x_{n} \in\left[l_{n}^{\prime} . . h_{n}^{\prime}\right] \cap D_{n}\right\rangle}
\end{gathered}
$$

where $l_{j}^{\prime}$ and $h_{j}^{\prime}$ are defined as in the LINEAR INEQUALITY 1 reduction rule with $l_{j}:=$ $\min \left(D_{j}\right)$ and $h_{j}:=\max \left(D_{j}\right)$.

Note that in this rule the domains are now arbitrary finite sets of integers. An analogous modification can be introduced for the case of the reduction rule for equality constraints.

In the case of a simple disequality constraint we use the DISEQUALITY 3 solving rule. So now, in contrast to the case of interval domains, an arbitrary element can be removed from a domain, not only the "boundary" one.

\subsection{Example: the $S E N D+M O R E=M O N E Y$ Puzzle}

We now illustrate use of the above rules for linear constraints over interval and finite domains by analyzing in detail the well-known SEND + MORE $=M O N E Y$ puzzle. Recall that this puzzle calls for a solution of the equality constraint

$$
\begin{aligned}
& 1000 \cdot S+100 \cdot E+10 \cdot N+D \\
+ & 1000 \cdot M+100 \cdot O+10 \cdot R+E \\
= & 10000 \cdot M+1000 \cdot O+100 \cdot N+10 \cdot E+Y
\end{aligned}
$$

together with 28 simple disequality constraints $x \neq y$ for $x, y \in\{S, E, N, D, M, O, R, Y\}$ where $x$ preceeds $y$ in the alphabetic order, and with the range [1..9] for $S$ and $M$ and the range [0..9] for the other variables.

Both in the CHIP system (see [25, page 143]) and in $\mathrm{ECL}^{i} \mathrm{PS}^{e}$ Version 3.5.2. the above CSP is internally reduced to the one with the following domain expressions:

$$
S=9, E \in[4 . .7], N \in[5 . .8], D \in[2 . .8], M=1, O=0, R \in[2 . .8], Y \in[2 . .8] .
$$

We now show how this outcome can be formally derived using the rules we introduced.

First, using the transformation rules for linear constraints we can transform the above equality to

$$
9000 \cdot M+900 \cdot O+90 \cdot N+Y-(91 \cdot E+D+1000 \cdot S+10 \cdot R)=0 .
$$


Applying the LINEAR EQUALITY reduction rule with the initial ranges we obtain the following sequence of new ranges:

$$
S=9, E \in[0 . .9], N \in[0 . .9], D \in[0 . .9], M=1, O \in[0 . .1], R \in[0 . .9], Y \in[0 . .9] .
$$

At this stage a subsequent use of the same rule yields no new outcome. However, by virtue of the fact that $M=1$ we can now apply the SIMPLE DISEQUALITY 3 solving rule to $M \neq O$ to conclude that $O=0$. Using now the facts that $M=1, O=0, S=9$, the solving rules SIMPLE DISEQUALITY 2 and 3 can be repeatedly applied to shrink the ranges of the other variables. This yields the following new sequence of ranges:

$$
S=9, E \in[2 . .8], N \in[2 . .8], D \in[2 . .8], M=1, O=0, R \in[2 . .8], Y \in[2 . .8] .
$$

Now five successive iterations of the LINEAR EQUALITY reduction rule yield the following sequences of shrinking ranges of $E$ and $N$ with other ranges unchanged:

$$
\begin{aligned}
& E \in[2 . .7], N \in[3 . .8], \\
& E \in[3 . .7], N \in[3 . .8], \\
& E \in[3 . .7], N \in[4 . .8], \\
& E \in[4 . .7], N \in[4 . .8], \\
& E \in[4 . .7], N \in[5 . .8],
\end{aligned}
$$

upon which the reduction process stabilizes. At this stage the solving rules for disequalities are not applicable either.

So using the reduction rules we reduced the original ranges to (6). The derivation, without counting the initial applications of the transformation rules, consists of 24 steps.

Using the SUBSTITUTION rule of Subsection 2.2 and obvious transformation rules that deal with bringing the equality constraints to the form (5), the original equality constraint gets reduced to

$$
90 \cdot N+Y-(91 \cdot E+D+10 \cdot R)=0 .
$$

Moreover, ten simple disequality constraints between the variables $E, N, D, R$ and $Y$ are still present.

Further progress can now be obtained only by employing a splitting rule. The behaviour of the ECL ${ }^{i} \mathrm{PS}^{e}$ finite domain solver is modelled by the ENUMERATION rule of Subsection 2.2 . It can be shown, by mimicking the $\mathrm{ECL}^{i} \mathrm{PS}^{e}$ execution, that, when the rules here presented are augmented by this rule, there exists a successful derivation for the original CSP representing the $S E N D+M O R E=M O N E Y$ puzzle. 


\subsection{Discussion}

This concludes our presentation of the proof rules that can be used to build a constraint solver for linear constraints over interval and finite domains. Such proof rules are present in one form or another within each constraint programming system that supports linear constraints over interval and finite domains.

It is worthwhile to mention that the reduction rules LINEAR INEQUALITY 1 and LINEAR EQUALITY are simple modifications of the reduction rule introduced in [9, page 306] that dealt with closed intervals of reals. Also, as pointed out to us by Lex Schrijver, these rules are instances of the cutting-plane proof technique used in linear programming (see e.g. [7, Section 6.7]).

\section{Building a Constraint Solver: an Example}

We now show how one can use our approach to define specific constraint solvers. By means of example consider the constraint exactly $(x, l, z)$ introduced in [26]. It states that for a list $l$ of variables ranging over some fixed domain exactly $x$ of its elements equal $z$. We assume that this primitive can be used with $x$ a variable ranging over a subset of natural numbers and with $z$ a variable with an unspecified domain. (This is not a restriction since we can augment the rules below with some natural variable introduction rules.) The exactly $(x, l, z)$ primitive is useful for dealing with scheduling problems.

Below we assume that $m>0, i \in[1 . . m]$ and that $\mathcal{D E}$ stands for a sequence of domain expressions involving the relevant variables. Further, given a set $D$ of natural numbers, we define

$$
D-1:=\{d-1 \mid d \in D, d>0\} .
$$

The behaviour of the exactly $(x, l, z)$ primitive is described by means of the following four rules:

$$
\begin{gathered}
\operatorname{EXACTLY} 1 \\
\frac{\left\langle\operatorname{exactly}\left(x,\left[y_{1}, \ldots, y_{m}\right], z\right) ; x \in D_{x}, \mathcal{D E}\right\rangle}{\left\langle\operatorname{exactly}\left(x,\left[y_{1}, \ldots, y_{m}\right], z\right) ; x \in D_{x}-\{d \mid d>m\}, \mathcal{D E}\right\rangle} \\
\frac{\langle\operatorname{EXACTLY} 2}{\left\langle\operatorname{exactly}\left(x,\left[y_{1}, \ldots, y_{m}\right], z\right) ; y_{i} \in D_{i}, z \in D_{z}, \mathcal{D E}\right\rangle}
\end{gathered}
$$

where $D_{i} \cap D_{z}=\emptyset$ and $m>1$,

$$
\text { EXACTLY } 3
$$

$$
\frac{\left\langle\operatorname{exactly}\left(x,\left[y_{1}, \ldots, y_{m}\right], z\right) ; x \in D_{x}, y_{i}=a, z=a, \mathcal{D E}\right\rangle}{\left\langle\operatorname{exactly}\left(u,\left[y_{1}, \ldots, y_{i-1}, y_{i+1}, \ldots, y_{m}\right], z\right), u=x-1 ; u \in D_{x}-1, x \in D_{x}, y_{i}=a, z=a, \mathcal{D E}\right\rangle}
$$




$$
\begin{gathered}
\operatorname{EXACTLY} \& \\
\frac{\left\langle\operatorname{exactly}\left(x,\left[y_{1}, \ldots, y_{m}\right], z\right) ; x=0, \mathcal{D E}\right\rangle}{\left\langle y_{1} \neq z, \ldots, y_{m} \neq z ; x=0, \mathcal{D E}\right\rangle}
\end{gathered}
$$

It is straightforward to see that these four rules are equivalence preserving. These rules, when augmented with a modification of the LINEAR EQUALITY rule for finite domains, DISEQUALITY 1,2 and 3 rules and the ENUMERATION rule, form a stand alone constraint solver.

Such a solver can be directly used for example to solve Latin square puzzles. (Recall that a Latin square of order $n$ is defined to be an $n \times n$ array made out of the integers $1,2, \ldots, n$ with the property that each of the $n$ symbols occurs exactly once in each row and exactly once in each column of the array.)

More importantly, the exactly $(x, l, z)$ primitive has been used to specify certain type of scheduling problems, such as the car sequencing problem (see [26]). Also, it can be used in turn to define other useful constraint primitives, such as the $\operatorname{atmost}(x, l, z)$ primitive of [26] that states that for a list $l$ of variables ranging over some fixed domain atmost $x$ of its elements equal $z$. To this end it suffices to adopt the following rule

$$
\frac{\left\langle\operatorname{atmost}(x, l, z) ; x \in D_{x}, \mathcal{D E}\right\rangle}{\left\langle\operatorname{exactly}(y, l, z), y \leq x ; y \in D_{x}, x \in D_{x}, \mathcal{D E}\right\rangle}
$$

and add an instance of the LINEAR INEQUALITY 2 rule that deals with simple inequalities of the form $y \leq x$.

\section{Conclusions}

\subsection{Summing up}

We presented here a proof theoretic framework that allows us to model computing using constraints.

In general, constraint programming consists of a generation of constraints and of solving them. Both phases can be intertwined. In our presentation we only concentrated on the latter aspect of constraint programming. To complete the picture the framework here presented should be combined with a specific "host" programming language from which the constraints can be generated. To model computing in such an amalgamated language the proof rules should be combined with transitions dealing with the program state. In such a constraint programing language one can distinguish two computation steps.

- If a "conventional" programming statement (such as a procedure call or a built-in in the logic programming framework, or an assignment or a WHILE loop in the imperative programming framework) is encountered, a usual transition is performed and the program state is modified accordingly. 
- If a constraint is encountered, it is added to the current set of constraints ("constraint store"). This addition is followed by a repeated application of the proof rules to the constraint store. The order of application of these rules is determined by some builtin scheduler (see the next subsection). The terminating condition depends on specific applications.

Further, in such an amalgamated language the interaction between the constraint store and the program state should be properly taken care of.

In the case of linear constraints on finite domains the deterministic proof rules are repeatedly applied until all constraints are solved or a CSP is generated that is closed under the applications of these rules. In the case of algebraic constraints on real intervals the proof rules are repeatedly applied until all constraints are solved or all intervals are smaller than some fixed in advance $\epsilon$.

In some constraint programming languages or in the case of some constraint solvers the splitting rules are not scheduled. Instead, their application is explicitly triggered by some programming construct or facility present in the language.

Let us compare now in more detail our approach to that of [6] and [10]. In [6] the proof rules are represented as rewrite rules in the programming language ELAN (for the most recent reference see [4]). ELAN allows one to define specific strategies that can be used to schedule these rewrite rules. We defer discussion of this aspect to the next subsection.

In constraint handling rules (CHRs) of [10] the rules manipulate constraints only, so the domains need to be encoded as unary constraints. This leads to a different than ours classification of rules according to which "propagation" means addition of redundant constraints. Further, these rules are not supposed to be used "stand alone" but rather to augment constraint logic programming that provides already a support for the "don't know" nondeterminism. So no splitting rules are available.

So the implementation of constraint solvers defined by the ELAN rules and by CHRs is automatically provided by the interpreter, respectively compiler of the language. In contrast, our approach is not geared towards direct implementability even though an implementation specified by such rules is pretty obvious. This allows us to be more abstract and permits us to express and analyze specific, domain dependent, constraint solvers in a simple way.

For example we can readily define proof rules that involve some auxiliary computations, such as the LINEAR INEQUALITY reduction rule of Subsection 3.2 and study formally properties of such rules (see Appendix). Such an analysis would be difficult to achieve if we had to reason about an encoding of these rules in a specific programming formalism.

\subsection{Control}

One of the issues conspicuously absent in our considerations is that of control. To draw the analogy with the "Algorithm = Logic + Control" slogan of [12], what we defined here is only the "Logic" part of constraint programming. The picture is completed once we can adequately deal with the "Control" part. 
In the proof theoretic framework here presented the issue of control enters the picture at three places. First, one needs to schedule the introduced proof rules. Second, one should be able to define which rules are to be scheduled. Finally, one needs some search strategy to traverse the generated proof trees in search for a successful derivation.

The scheduling of the proof rules could be done using a built-in strategy that combines scheduling of the deterministic rules by means of a generic chaotic iteration algorithm of [2] with the requirement that the applications of the splitting rules are delayed as much as possible. We noted in [2] that several constraint propagation algorithms employ in fact such a generic algorithm. Further, delay of the applications of the splitting rules prevents unnecessary creation of alternative branches and is a well-known and widely used heuristic. So such a "hard-wired" strategy seems perfectly reasonable.

In contrast, we think that both the selection of specific proof rules and the search strategies for traversing the proof trees should be programmable. What we need here is a programming notation that could allow us to define most common search strategies in a simple way. One possibility would be to use ELAN that, as already mentioned, allows one to define various search strategies. [6] showed how several of them, such as forward checking and various forms of look ahead, can be implemented in ELAN. We believe that more work is needed to see whether other strategies such as backjumping can be expressed in ELAN, as well.

Another work that should be mentioned here is [20] where it is shown how the concept of so-called computation spaces can be used to program in a simple way various search strategies in the programming language $\mathrm{Oz}$ (see [22]).

\section{Acknowledgements}

We would like to thank Eric Monfroy for numerous discussions on the subject of this paper and Carlos Castro, Martin Henz, Lex Schrijver, Gerhard Wetzel and an anonymous referee for providing useful comments on a draft of this paper.

\section{References}

[1] J. H. Andrews. Foundational issues in implementing constraint logic programming systems. Science of Computer Programming, 25(2 \& 3):117-148, 1995.

[2] K. R. Apt. From chaotic iteration to constraint propagation. In P. Degano, R. Gorrieri, and A. Marchetti-Spaccamela, editors, Proceedings of the 24th International Colloquium, ICALP '97, volume 1256 of Lecture Notes in Computer Science, pages 36-55, New York, 1997. Springer-Verlag. Invited Lecture.

[3] N. Bleuzen Guernalec and A. Colmerauer. Narrowing a $2 n$-block of sortings in $O(n \log (n)$. In G. Smolka, editor, Proceedings of the 3rd International Conference on Constraint Programming (CP97), Lecture Notes in Computer Science, vol. 1330, pages 2-16, Berlin, 1997. Springer-Verlag. 
[4] Peter Borovanský, Claude Kirchner, and Hélène Kirchner. A functional view of rewriting and strategies for a semantics of ELAN. In The Third Fuji International Symposium on Functional and Logic Programming, Kyoto, Japan, April 1998.

[5] Y. Caseau and F. Laburthe. Introduction to the CLAIRE programming language. Technical report, Departement Mathématiques et Informatique, Ecole Normale Supérieure, Paris, France, 1996.

[6] C. Castro. Building constraint satisfaction problem solvers using rewrite rules and strategies. Fundamenta Informaticae, 1998. This issue.

[7] W.J. Cook, W.H. Cunningham, W.R. Pulleyblank, and A. Schrijver. Combinatorial Optimization. John Wiley \& Sons, Inc., 1998.

[8] J. Darlington and Y. Guo. Constraint logic programming in the sequent calculus. In F. Pfenning, editor, Logic Programming and Automated Reasoning, volume 822 of Lecture Notes in Computer Science, pages 200-214, New York, 1994. Springer-Verlag.

[9] Ernest Davis. Constraint propagation with interval labels. Artificial Intelligence, 32(3):281331, July 1987.

[10] Thom Frühwirth. Constraint Handling Rules. In Andreas Podelski, editor, Constraint Programming: Basics and Trends, LNCS 910, pages 90-107. Springer-Verlag, 1995. (Châtillonsur-Seine Spring School, France, May 1994).

[11] J. Jaffar and M.J. Maher. Constraint logic programming: A survey. Journal of Logic Programming, 19,20:503-581, 1994.

[12] R. A. Kowalski. Algorithm = logic + control. Communications of the ACM, 22(7):424-435, 1979.

[13] R. A. Kowalski, F. Toni, and G. Wetzel. Executing suspended logic programs. Fundamenta Informaticae, 1998. This issue.

[14] O. Lhomme. Consistency techniques for numeric CSPs. In Proceedings of the International Joint Conference on Artificial Intelligence (IJCAI-93), pages 232-238, 1993.

[15] Alan Mackworth. Consistency in networks of relations. Artificial Intelligence, 8(1):99-118, 1977.

[16] D. McAllester. Truth maintenance. In AAAI-90: Proceedings 8th National Conference on Artificial Intelligence, pages 1109-1116, 1990.

[17] K. McAloon and C. Tretkoff. 2LP: Linear programming and logic programming. In P. Van Hentenryck and V. Saraswat, editors, Principles and Practice of Constraint Programming, pages 101-116. MIT Press, 1995.

[18] R. Mohr and G. Masini. Good old discrete relaxation. In Y. Kodratoff, editor, Proceedings of the 8th European Conference on Artificial Intelligence (ECAI), pages 651-656. Pitman Publishers, 1988.

[19] J.-C. Regin. A filtering algorithm for constraints of difference in CSPs. In AAAI-94: Proceedings of the 12th National Conference on Artificial Intelligence, pages 362-367, 1994.

[20] C. Schulte. Programming constraint inference engines. In G. Smolka, editor, Proceedings of the 3rd International Conference on Constraint Programming (CP97), Lecture Notes in Computer Science, vol. 1330, pages 519-533, Berlin, 1997. Springer-Verlag. 
[21] S. M. Sieber, Y. Schabes, and F. C. N. Pereira. Principles and implementation of deductive parsing. Journal of Logic Programming, 24(1 \& 2):3-36, 1995.

[22] G. Smolka. The Oz programming model. In Jan van Leeuwen, editor, Computer Science Today, Lecture Notes in Computer Science, vol. 1000, pages 324-343. Springer-Verlag, Berlin, 1995.

[23] G. Smolka. Problem solving with constraints and programming. ACM Computing Surveys, 28(4es):, 1996. Electronic Section.

[24] M. H. van Emden. Value constraints in the CLP scheme. Constraints, 2(2):163-184, 1997.

[25] P. van Hentenryck. Constraint Satisfaction in Logic Programming. Logic Programming Series, The MIT Press, Cambridge, MA, 1989.

[26] Pascal Van Hentenryck, Helmut Simonis, and Mehmet Dincbas. Constraint satisfaction using constraint logic programming. Artificial Intelligence, 58:113-159, 1992.

[27] Van Hentenryck, Saraswat \& et al. Strategic directions in constraint programming. $A C M$ Computing Surveys, 28(4):701-726, 1996.

[28] G. Wetzel, R. Kowalski, and F. Toni. A theorem-proving approach to CLP. In A. Krall and U. Geske, editors, 11th Workshop on Logic Programming. GMD-Studien Nr. 270, TU Wien, 1995.

\section{Appendix: a Characterization of the LINEAR EQUALITY Rule}

The rules we introduced in Section 3 seem somewhat arbitrary. After all, using them we cannot even solve the CSP $\langle x+y=10, x-y=0 ; x \in[0 . .10], y \in[0 . .10]\rangle$ as no application of the LINEAR EQUALITY rule of Subsection 3.2 to this CSP is relevant.

The point is that the domain reduction rules like the LINEAR EQUALITY rule are in some sense "orthogonal" to the rules that deal with algebraic manipulations (that can be expressed as transformation rules): their aim is to reduce the domains and not to transform constraints. So it makes sense to clarify what these rules actually achieve. This is the aim of this section. By means of example, we concentrate here on the LINEAR EQUALITY rule.

To analyze it it will be useful to consider first its counterpart that deals with the intervals of reals. This rule is obtained from the LINEAR EQUALITY rule by deleting from the definitions of $l_{j}^{\prime}$ and $h_{j}^{\prime}$ the occurrences of the functions \lceil\rceil and \lfloor\rfloor . So this rule deals with an equality constraint in the form

$$
\sum_{i \in P O S} a_{i} x_{i}-\sum_{i \in N E G} a_{i} x_{i}=b
$$

where

- $a_{i}$ is a positive integer for $i \in P O S \cup N E G$,

- $x_{i}$ and $x_{j}$ are different variables for $i \neq j$ and $i, j \in P O S \cup N E G$,

- $b$ is an integer, 
and intervals over reals.

In what follows for two reals $r_{1}$ and $r_{2}$ we denote by $\left[r_{1}, r_{2}\right]$ the closed interval of real line bounded by $r_{1}$ and $r_{2}$. So $\pi \in[3,4]$ while $[3 . .4]=\{3,4\}$.

The rule in question has the following form:

\section{R-LINEAR EQUALITY}

$$
\frac{\left\langle\sum_{i \in P O S} a_{i} x_{i}-\sum_{i \in N E G} a_{i} x_{i}=b ; x_{1} \in\left[l_{1} . . h_{1}\right], \ldots, x_{n} \in\left[l_{n} . . h_{n}\right]\right\rangle}{\left\langle\sum_{i \in P O S} a_{i} x_{i}-\sum_{i \in N E G} a_{i} x_{i}=b ; x_{1} \in\left[l_{1}^{r} . . h_{1}^{r}\right], \ldots, x_{n} \in\left[l_{n}^{r} . . h_{n}^{r}\right]\right\rangle}
$$

where for $j \in P O S$

$$
l_{j}^{r}:=\max \left(l_{j}, \gamma_{j}\right), h_{j}^{r}:=\min \left(h_{j}, \alpha_{j}\right)
$$

and for $j \in N E G$

$$
l_{j}^{r}:=\max \left(l_{j}, \beta_{j}\right), h_{j}^{r}:=\min \left(h_{j}, \delta_{j}\right) .
$$

Recall that $\alpha_{j}, \beta_{j}, \gamma_{j}$ and $\delta_{j}$ are defined in Subsection 3.2. In particular, recall that

$$
\alpha_{j}=\frac{b-\sum_{i \in P O S-\{j\}} a_{i} l_{i}+\sum_{i \in N E G} a_{i} h_{i}}{a_{j}}
$$

and

$$
\gamma_{j}:=\frac{b-\sum_{i \in P O S-\{j\}} a_{i} h_{i}+\sum_{i \in N E G} a_{i} l_{i}}{a_{j}}
$$

It is straightforward to see that the $\mathcal{R}$-LINEAR EQUALITY rule, when interpreted over the intervals of reals, is also equivalence preserving.

To characterize the $\mathcal{R}$-LINEAR EQUALITY rule we use the following notion introduced in [18]. The original definition for binary constraints is due to [15].

\section{Definition 5.1.}

- A constraint $C$ is called arc-consistent if for every variable of it each value in its domain participates in a solution to $C$.

- A CSP is called arc-consistent if every constraint of it is.

We now prove the following result.

\section{Theorem 5.1.}

(i) The conclusion of the $\mathcal{R}-L I N E A R$ EQUALITY rule is either failed or arc consistent.

(ii) In the case of a single linear equality constraint the $\mathcal{R}$-LINEAR EQUALITY rule is idempotent, that is a CSP is closed under the application of this rule after one iteration.

(iii) In the case of more than one linear equality constraint the $\mathcal{R}$-LINEAR EQUALITY rule can yield an infinite derivation. 


\section{Proof:}

(i) Assume that the conclusion of the R-LINEAR EQUALITY rule is not failed. Fix $j \in P O S$. We have both

$$
\sum_{i \in P O S-\{j\}} a_{i} l_{i}+a_{j} \alpha_{j}-\sum_{i \in N E G} a_{i} h_{i}=b
$$

and

$$
\sum_{i \in P O S-\{j\}} a_{i} h_{i}+a_{j} \gamma_{j}-\sum_{i \in N E G} a_{i} l_{i}=b
$$

Hence for any $\alpha$

$$
\alpha\left(\sum_{i \in P O S-\{j\}} a_{i}\left(l_{i}-h_{i}\right)+a_{j}\left(\alpha_{j}-\gamma_{j}\right)-\sum_{i \in N E G} a_{i}\left(h_{i}-l_{i}\right)\right)=0
$$

so for any $\alpha$

$$
\sum_{i \in P O S-\{j\}} a_{i}\left(h_{i}+\alpha\left(l_{i}-h_{i}\right)\right)+a_{j}\left(\gamma_{j}+\alpha\left(\alpha_{j}-\gamma_{j}\right)\right)-\sum_{i \in N E G} a_{i}\left(l_{i}+\alpha\left(h_{i}-l_{i}\right)\right)=b .
$$

By the definition of $l_{j}^{r}$ and $h_{j}^{r}$ we have $\left[l_{j}^{r}, h_{j}^{r}\right] \subseteq\left[\gamma_{j}, \alpha_{j}\right]$. By the initial assumption the interval $\left[l_{j}^{r}, h_{j}^{r}\right]$ is non-empty.

Take now some $d \in\left[l_{j}^{r}, h_{j}^{r}\right]$. Next, take $\alpha$ such that $\gamma_{j}+\alpha\left(\alpha_{j}-\gamma_{j}\right)=d$, that is

$$
\alpha:=\frac{d-\gamma_{j}}{\alpha_{j}-\gamma_{j}}
$$

and choose the solution to $\sum_{i \in P O S} a_{i} x_{i}-\sum_{i \in N E G} a_{i} x_{i}=b$ determined by $\alpha$ and (7).

By the choice of $d$ and the fact that $\left[l_{j}^{r}, h_{j}^{r}\right]$ is non-empty, $\alpha$ is well-defined and $0 \leq \alpha \leq 1$. Hence this solution to $\sum_{i \in P O S} a_{i} x_{i}-\sum_{i \in N E G} a_{i} x_{i}=b$ lies in the ranges $\left[l_{i} . . h_{i}\right]$ with $i \in[1 . . n]$. But the $\mathcal{R}$-LINEAR EQUALITY rule is equivalence preserving, so this solution also lies in the ranges $\left[l_{i}^{r} . . h_{i}^{r}\right]$ with $i \in[1 . . n]$. This proves the claim.

(ii) Straightforward by (i) as the domains of an arc consistent CSP cannot be reduced without losing equivalence.

(iii) The following example is due to [9, page 304]. Take $\langle x=y, x=2 y ; x \in[0,100], y \in[0,100]\rangle$ and consider a derivation in which the constraints are selected in an alternating fashion. This derivation is easily seen to be infinite.

Let us mention here that property (i) generalizes a corresponding result stated in $[9$, page $326]$ for the more limited case of so-called unit coefficient constraints.

In contrast, the LINEAR EQUALITY rule behaves differently. First, it is not idempotent even in the case of a single linear equality - it just suffices to see the example at the end of Subsection 3.2. Second, in the case of several linear equality constraints its repeated use always 
terminates (due to the fact that the domains are finite) and yields a CSP that is closed under the applications of this rule (by the fact that it is equivalence preserving).

Further, the example at the end of Subsection 3.2 also shows that a CSP closed under the applications of this rule does not need to be arc consistent. So we need another notion to characterize CSP's closed under the applications of this rule.

First, we introduce the following terminology.

\section{Definition 5.2.}

- By an ICSP we mean a CSP the domains of which are intervals of reals or integers.

- A constraint $C$ on a non-empty sequence of variables, the domains of which are intervals of reals or integers, is called bound consistent if for every variable of it each of its two bounds participates in a solution to $C$.

- An ICSP is called bound consistent if every constraint of it is.

This notion is motivated by a similar concept introduced in [14] in the case of constraints on reals. Note that if a constraint with interval domains is bound consistent, then both the constraint and each of its intervals is non-empty.

Denote now by LINEQ an ICSP all constraints of which are linear equalities of the form (5) of Subsection 3.2 and discussed above.

Definition 5.3. Consider a LINEQ $\phi$. Let $\phi^{r}$ denote the CSP obtained from $\phi$ by replacing each integer domain $[l . . h]$ by the corresponding interval $[l, h]$ of reals. We say that $\phi$ is interval consistent if $\phi^{r}$ is bound consistent.

So $\phi$ is interval consistent if for every constraint $C$ of it the following holds: for every variable of $C$ each of its two bounds participates in a solution to $\phi^{r}$.

For example, the CSP $\langle 3 x-5 y=4 ; x \in[3 . .9], y \in[1 . .4]\rangle$ of Subsection 3.2 is bound consistent as both $x=3, y=1$ and $x=8, y=4$ are solutions of $3 x-5 y=4$.

In contrast, the CSP $\phi:=\langle 2 x+2 y-2 z=1 ; x \in[0 . .1], y \in[0 . .1], z \in[0 . .1]\rangle$ is clearly not bound consistent but it is interval consistent. Indeed, the equation $2 x+2 y-2 z=1$ has three solutions in the unit cube formed by the real unit intervals for $x, y$ and $z:(0,0.5,0),(1,0,0.5)$ and $(0.5,1,1)$. So each bounds participates in a solution to $\phi^{r}:=\langle 2 x+2 y-2 z=1 ; x \in[0,1], y \in$ $[0,1], z \in[0,1]\rangle$.

The following result now characterizes the outcome of a repeated application of the LINEAR EQUALITY rule.

Theorem 5.2. Consider a LINEQ $\phi$ that is closed under the applications of the LINEAR EQUALITY rule. Then $\phi$ is either failed or interval consistent.

\section{Proof:}

The intervals of reals corresponding to the integer intervals in the conclusion of the $L I N$ $E A R E Q U A L I T Y$ rule are respectively smaller than those in the conclusion of the $\mathcal{R}$-LINEAR EQUALITY rule. So the assumption implies that $\phi^{r}$ is closed under the applications of the $\mathcal{R}$-LINEAR EQUALITY rule.

Assume now that $\phi$ is not failed. Then $\phi^{r}$ is not failed either. By Theorem $5.1 \phi^{r}$ is arc consistent, so a fortiori bound consistent. 
The example preceding the above theorem shows that interval consistency cannot be replaced here by bound consistency. In other words, to characterize the LINEAR EQUALITY rule it is needed to resort to a study of solutions over reals.

Consider now a LINEQ CSP $\phi$ and a derivation that consists solely of the applications of the LINEAR EQUALITY rule. As noticed before it is finite. Let $\psi$ be the final LINEQ CSP. So $\psi$ is closed under the applications of the LINEAR EQUALITY rule. By Theorem $5.2 \psi$ is failed or interval consistent.

Assume now that $\phi$ is not failed. Using the results of [2] (more specifically Theorem 13 on page 47 ), we can then characterize $\psi$ as the largest interval consistent LINEX CSP that is smaller than $\phi$ and equivalent to it. (The equivalence of $\psi$ and $\phi$ is due to the fact that the LINEAR EQUALITY rule is equivalent preserving.) This paper also shows how the applications of the LINEAR EQUALITY rule can be scheduled in a meaningful way by means of a generic chaotic iteration algorithm. The details should be clear to any reader of this paper but a detailed exposition here would take us too far afield.

Similar characterizations results can be envisaged for other proof systems characterizing specific type of constraints. Such characterizations have been considered in the literature albeit not in a proof theoretical framework.

Let us cite just two examples. In [19] the constraint primitive alldistinct $\left(\left[x_{1}, \ldots, x_{n}\right]\right)$ that states that the listed variables are all distinct was characterized using the arc consistency notion.

Next, in [3] the constraint primitive $\operatorname{sort}\left(\left[x_{1}, \ldots, x_{n}\right],\left[y_{1}, \ldots, y_{n}\right]\right)$ was characterized using the bound consistency notion. This constraint states that the second list is the sorted version of the first list; the variables are assumed to range over intervals.

In each case an algorithm was provided that reduces the domains of the constraint under consideration so that the appropriate local consistency notion is satisfied. On a sufficiently abstract level these algorithms can be explained by means of reduction rules.

We believe that such results allow us both to clarify and to characterize existing constraint solvers and to look for new ones. In this respect an interesting question is in what sense the rules of Section 4 characterize the exactly $(x, l, z)$ primitive. 